\title{
Impacts socio-économiques et environnementaux de la promotion des ouvrages ECOSAN dans le développement de la commune de Sèmè-Podji au sud du Bénin
}

\author{
Offin Lié Rufin AKIYO ${ }^{1 *}$, Alix Servaix AFOUDA ${ }^{1}$, Ibouraïma YABI ${ }^{2}$, \\ Julien ADOUNKPE ${ }^{3}$ et Benoît N'BESSA ${ }^{2}$ \\ ${ }^{I}$ Départements de Sociologie-Anthropologie et de Géographie, FLASH/Université de Parakou, \\ République du Bénin. \\ ${ }^{2}$ Département de Géographie, FLASH/Université d'Abomey-Calavi, République du Bénin. \\ ${ }^{3}$ Département de l'Aménagement et de la Gestion de l'Environnement/FSA Université d'Abomey-Calavi, \\ République du Bénin. \\ *Auteur correspondant, E-mail : rufinakiyo@yahoo.fr; Tel: +22997476110 ; +22994207086.
}

\section{RESUME}

Les populations de la commune de Sèmè-Podji, située dans le département de l'Ouémé au sud du Bénin, sont de plus en plus confrontées au problème d'hygiène et d'assainissement dû à l'absence de toilettes et de services d'assainissement adaptés. Cette situation est la cause de plusieurs pathologies qui sévissent dans le milieu avec pour conséquences, l'élévation du taux de morbidité et une dégradation très poussée de l'environnement. Pour parer à ces problèmes, cette localité, avec le soutien de certains partenaires techniques et financiers notamment le Centre Africain pour l'Eau Potable et l'Assainissement à faible coût (CREPA) Bénin a opté depuis quelques années pour la promotion des latrines d'assainissement écologique (ECOSAN) peu coûteuses. Ces latrines et urinoirs sont des fosses étanches ou non d'aisance, semi-enterrées ou surélevées avec la particularité de séparer les fèces et les urines en vue de faciliter leur traitement pour une réutilisation saine. L'objectif de la présente recherche est de montrer l'importance de l'utilisation de ce système d'éco assainissement dans la réduction des maladies d'origine hydro fécale, l'acquisition à faible coût d'éléments fertilisants et moins nocifs à l'instar des engrais chimiques très coûteux qui polluent l'environnement et causent de grands préjudices aux populations. Pour atteindre cet objectif, l'entretien individuel, le questionnaire, le Focus Group et l'observation directe ont servi de base méthodologique. Les résultats obtenus à partir de ces outils ont été analysés grâce au modèle SWOT. De ces résultats obtenus, il se révèle la nécessité de promouvoir la construction de cet ouvrage dans tous les villages de la commune de Sèmè-Podji pour une amélioration quantitative et qualitative des conditions de vie des populations. Pour ce faire, un surplus d'efforts est nécessaire aussi bien au niveau de l'Etat que des collectivités locales sur les plans financier et communicationnel en vue de l'accroissement du nombre desdits ouvrages et de leur appropriation culturelle par certains habitants encore réticents.

(C) 2013 International Formulae Group. All rights reserved.

Mots clés : Sèmè-Podji, environnement, pollution, assainissement écologique, excréta. 


\section{INTRODUCTION}

L'eau, l'hygiène et l'assainissement, tout comme l'éducation et la santé, constituent des facteurs fondamentaux de lutte contre la pauvreté dans le monde (PNUD, 2006). Mais, force est de constater que de nos jours, près de 1,1 milliard d'êtres humains vivant dans des pays en développement ne peuvent accéder à l'eau de manière appropriée et 2,6 milliards de personnes n'ont pas accès à l'assainissement de base (PNUD, 2006). De même, contrairement à l'approvisionnement en eau, les problèmes relatifs à l'assainissement et à la gestion des excréta humains sont des domaines qui n'ont pas reçu la même attention. Au Bénin, à peine $4 \%$ des ménages se lavent les mains à l'eau et au savon avant de manger ou après les selles, $17 \%$ des ménages évacuent correctement leurs déchets, $62 \%$ ne disposent pas d'ouvrages adéquats d'évacuation des excréta et $37 \%$ n'ont pas accès à l'eau potable dans les campagnes (EDS III, 2006). A toutes ces préoccupations environnementales, s'ajoute le péril fécal qui demeure un problème de santé publique dans les villes et campagnes (CREPA, 2003). Ainsi, les problèmes d'accès à l'eau et à l'assainissement sont récurrents. Dans les villes tout comme dans les campagnes, l'accès des populations à l'assainissement est une préoccupation. Cette situation engendre une morbidité importante (diarrhées, maux de ventre, etc.) et une dégradation de l'environnement dans lequel les populations vivent et élèvent leurs enfants (Akiyo, 2012). Notons que la plus grande partie de cette pollution, qui conduit à des taux élevés de morbidité, de malnutrition et de létalité, est surtout due à l'absence de toilettes et de services d'assainissement adaptés.

En effet, dans le souci de faire face aux nombreux défis du millénaire, la Communauté Internationale s'est réunie en septembre 2000 à l'occasion du Sommet du millénaire des Nations Unies et s'est fixée huit (08) objectifs dits «Objectifs du Millénaire pour le Développement (OMD)». Au nombre de ceux-ci figure la question récurrente de l'eau et de l'assainissement dont la résolution permettra de réduire d'ici 2015 , le taux de mortalité dans les villes et campagnes. Face à cette réalité, la Représentation Nationale du CREPA-BENIN dans sa stratégie de prise en compte de ces paramètres environnementaux a inscrit dans ses programmes, la promotion de l'assainissement écologique (ECOSAN). Après plusieurs années d'essai et à miparcours de la phase de dissémination, il urge d'apprécier les impacts de l'appropriation de cet ouvrage d'assainissement dans la commune de Sèmè-Podji. C'est dans cette optique que s'inscrit la présente thématique.

Dans un premier temps, il s'agira donc d'apprécier le niveau de connaissance de l'approche ECOSAN au niveau des bénéficiaires et d'étudier les impacts socioéconomiques et environnementaux de cet ouvrage d'assainissement dans la commune de Sèmè-Podji en vue de proposer quelques approches solutions.

Quel est le matériel exploité et l'approche méthodologique utilisée dans le cadre de cette étude ?

\section{MATERIEL ET METHODES \\ Matériel}

Dans le cadre de cette étude, nous avons fait usage de plusieurs matériels notamment: une carte sommaire de reconnaissance du cadre d'étude (Figure 1), des fonds de cartes tirés de la carte générale du Bénin IGN mise à jour en 1992 et de l'Atlas monographique de l'Ouémé, un appareil photo numérique pour les prises de vue, les fiches, les images satellitaires LandSat ETM janvier 1996 Novembre 2008 et enfin un appareil Global Positionning System (GPS) pour prendre les coordonnées géographiques (latitude et longitude) des différents ouvrages réalisés. 


\section{Données}

Dans le cadre de la présente étude, plusieurs techniques et outils de collecte de données ont été utilisés. Les données collectées sont à la fois qualitatives et quantitatives. Les données qualitatives ont permis d'apprécier l'importance des ouvrages ECOSAN dans la protection de l'environnement et la promotion du développement durable au niveau de la Commune de Sèmè-Podji. Elles ont aussi servi à identifier le système d'évacuation des excréta, le degré d'utilisation des latrines ECOSAN par les ménages bénéficiaires des ouvrages, l'entretien des récipients de stockage d'urine, la perception du système ECOSAN par les bénéficiaires, le devenir des produits issus des latrines ECOSAN. Quant aux données statistiques, elles ont été recueillies au niveau des familles et institutions (hôpital, école) bénéficiant de cet ouvrage d'hygiène et d'assainissement. En effet, les informations collectées auprès de ces cibles sont relatives à l'état des ouvrages et à leur niveau de gestion.

\section{Technique d'échantillonnage et taille de l'échantillon}

Dans ce cadre, tous les ménages bénéficiaires des ouvrages ECOSAN et les institutions (hôpital, école, etc..) ont été pris en compte au niveau des six arrondissements composant la commune en vue de mieux apprécier les pratiques actuelles des populations en matière d'hygiène et d'assainissement (état des ouvrages) et d'évaluer les risques de maladies hydrofécales qu'elles encourent ainsi que l'acceptabilité de ce système amélioré d'assainissement mis en place par CREPA BENIN.

Au total, 47 familles, 4 centres de santé et 12 écoles primaires ont été pris en compte dans le cadre de cette étude.

\section{Population d'enquête}

La population cible concernée par cette enquête est constituée entre autres d'acteurs tels que: les chefs d'arrondissements, les chefs de quartiers, les notables, les chefs religieux, les agents des Ministères en charge de l'Environnement et de la Santé, du CREPA, de la Mairie de SèmèPodji, les ménages bénéficiaires, les membres d'associations de développement, les ONGs, les sociétés d'assainissement, etc.

Le critère sur le quel repose le choix raisonné de ces personnes interrogées est relatif à la masse et à la qualité d'informations qu'elles sont susceptibles de fournir sur le sujet d'étude.

\section{Outils et techniques de collecte des données}

L'accent a été mis sur l'importance des ouvrages ECOSAN dans la protection de l'environnement, la prévention des maladies hydro fécales, le nombre de bénéficiaires desdits ouvrages, l'entretien des récipients de stockage d'urine, la perception du système ECOSAN par les bénéficiaires, le devenir des produits issus des latrines ECOSAN au niveau de la Commune de Sèmè-Podji. A cet effet, les différentes données ont été collectées dans les villages des arrondissements de Djrègbé, d'Epkè, de Sèmè-Podji, d'Aholouyèmè, d'Agblangandan et de Tohouè abritant ces ouvrages d'assainissement. Dans ce cadre, nous avons donc recouru aux techniques cidessous :

$>$ l'entretien individuel réalisé à l'aide d'un guide d'entretien avec les chefs de villages, les chefs d'arrondissements, les chefs de quartiers, les notables, les chefs religieux ;

$>$ le focus group réalisé avec les différents bénéficiaires des ouvrages ECOSAN et plus précisément les bénéficiaires des produits recyclés, les membres d'associations de développement, des sociétés d'assainissement, les commerçants, les ménages, les institutions ; 
$>$ l'administration de questionnaire à certaines personnes cibles concernées par cette étude notamment les agents, d'hygiène, de CREPA Bénin, et des Ministères en charge de l'environnement et de la santé ;

$>$ l'observation directe d'un certain nombre de paramètres sociaux relatifs au système d'évacuation des excréta, au degré d'utilisation des latrines ECOSAN par les ménages bénéficiaires, à l'entretien des récipients de stockage d'urine.

La collecte des données statistiques s'est effectuée au niveau plusieurs structures et institutions de recherche comme l'Institut
National de la Statistique et de l'Analyse Economique (INSAE), les Ministères en charge de l'Environnement et de la Santé, le Centre Régional d'Eau Potable et Assainissement à faible coût (CREPA).

\section{Traitement des données}

Après cette étape, le dépouillement des fiches d'enquête a été effectué de façon manuelle. Quant aux informations quantitatives, notons qu'elles ont été traitées à l'aide des logiciels Word et Excel. Enfin, le traitement des données cartographiques a été effectué à l'aide du logiciel Arc View.

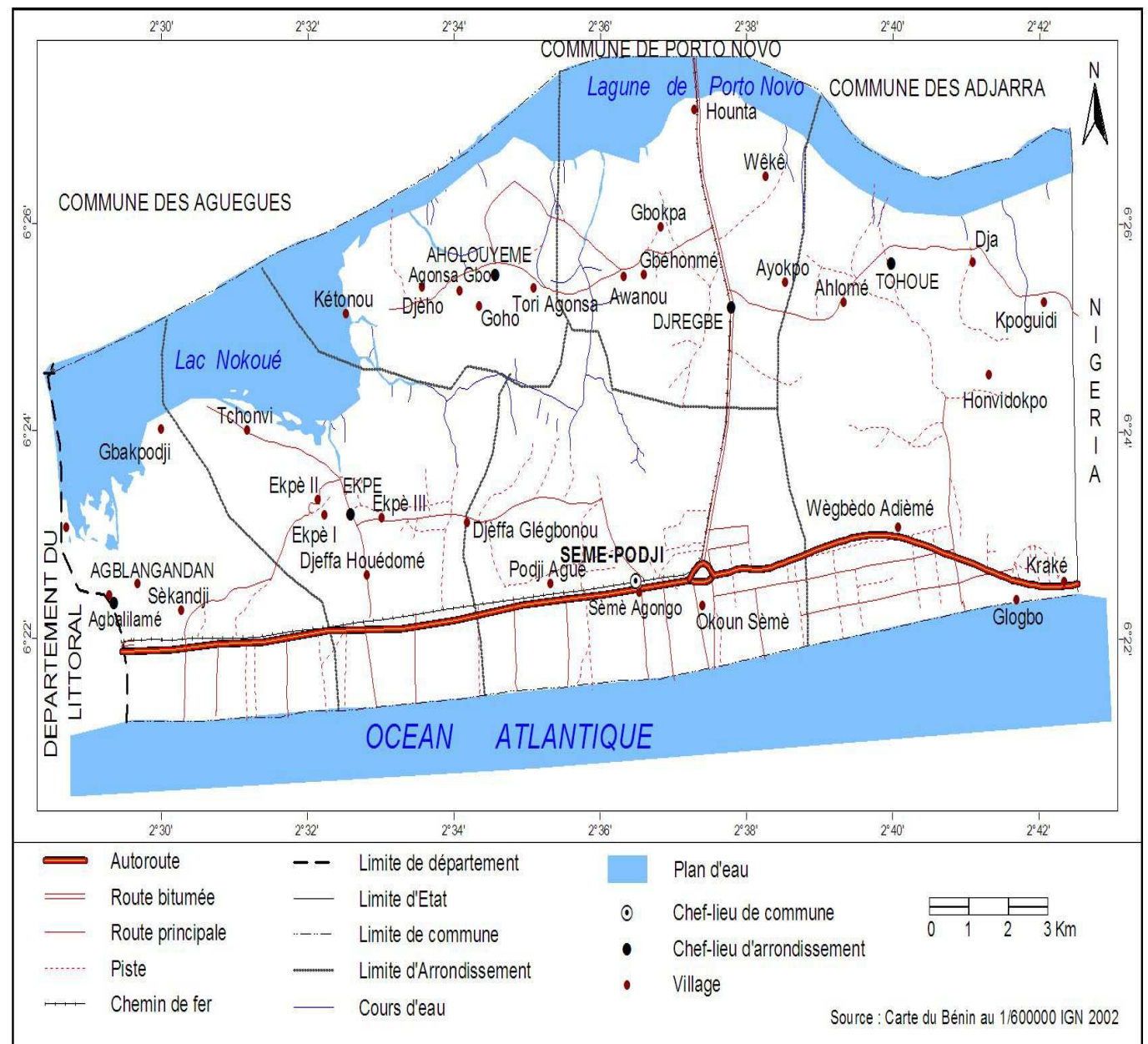

Figure 1 : La commune de Sèmè-Podji dans le département de l'Ouémé et ses arrondissements. Source : CENATEL, 2013. 


\section{Modèle d'analyse}

La méthode SWOT a été utilisée pour faire le diagnostic du milieu. En effet, SWOT (Strenght $=$ Forces/Atouts, Weaknesses $=$ Faiblesses/Contraintes, Opportunities = Opportunités, Threats $=$ Menaces) est un modèle d'analyse permettant de déterminer les options stratégiques envisageables au niveau d'un domaine. Elle a permis d'analyser les forces en présence dans le milieu d'étude, les opportunités dont il dispose, les menaces dont il faudra tenir compte dans l'assainissement de la Commune de Sèmè-Podji et enfin, les faiblesses ou contraintes des ouvrages ECOSAN dans le milieu et qui méritent une attention particulière. Elle intègre aussi bien les facteurs internes qu'externes de la perception du système ECOSAN par les populations de Sèmè-Podji. Ce modèle est utilisé pour mettre en exergue les différentes forces et faiblesses que présente la promotion d'une telle infrastructure dans ladite localité.

Quels sont les différents résultats obtenus à partir de cet arsenal de matériel et méthodes utilisés?

\section{RESULTATS}

Présentation du cadre d'étude Situation géographique et administrative

La commune de Sèmé-Podji est située dans le département de l'Ouémé, au Sud-Est de la République du Bénin sur la côte Atlantique entre $6^{\circ} 22^{\prime}$ et $6^{\circ} 28^{\prime}$ de latitude Nord et $2^{\circ} 28^{\prime}$ et $2^{\circ} 43^{\prime}$ de longitude Est. Elle s'étend sur une superficie de $218 \mathrm{Km}^{2}$, soit environ $0.19 \%$ de celle de la République du Bénin. Sèmé-Podji est limitée au Nord par la commune de Porto-Novo et des Aguégués, au Sud par l'Océan Atlantique, à l'Est par la République Fédérale du Nigéria et à l'Ouest par la commune de Cotonou.

Sur le plan administratif, la commune compte six (06) arrondissements subdivisés en trente quatre (34) villages et quatre (04) quartiers de villes. Le chef lieu est Podji Agué.

Ce milieu d'étude qui vient d'être décrit est illustré par la Figure 1.

Peuplement et démographie de Sèmè-Podji

A l'instar des autres communes du département de l'Ouémé, le peuplement de Sèmé-Podji est le résultat de différents courants migratoires. Il s'agit de ceux d'Alladanou, des Seto, puis les Xwla. Au troisième Recensement Général de la Population et de l'Habitat (RGPH) de 2002, cette commune comptait 115238 habitants. Cette population est caractérisée par la forte dominance de jeunes. En effet, $63 \%$ de la population a moins de 25 ans alors que celle âgée de plus de 55 ans ne représente que $5,16 \%$. C'est aussi une population en légère majorité féminine avec $51,46 \%$ de femmes. (RGPH3 de 2002).

\section{Relief et sol}

Sèmè-Podji est une plaine côtière encastrée dans un complexe de masses d'eau (Océan Atlantique, lagune de Porto-Novo, fleuve Ouémé et lac Nokoué). Il présente un relief très bas où se développe par endroits des activités agricoles et de la diversité des masses d'eau. La superficie cultivable fait $39,5 \%$ de la superficie totale de la commune. De plus, de par sa position topographique, la commune de Sèmè-Podji ne dispose que de sols résultant du lessivage ou de la sédimentation. Ces sols sont pour la plupart hydromorphes et très pauvres en éléments nutritifs et en matériaux organiques, notamment en base, azote et phosphore, mais riches en dioxyde de silicium avec quelques éléments de sol ferrugineux de type tropical. D'où l'importance de la promotion du système ECOSAN pour un meilleur enrichissement en nutriments naturels desdits sols.

\section{Végétation et climat}

La zone de Sèmè-Podji appartient au secteur phyto géographique guinéen côtier à végétation rase, clairsemée, formée 
essentiellement d'halophytes. La végétation naturelle, constituée d'arbustes et d'arbrisseaux denses à dominance de Zanthoxylum zanthoxylö̈des, Chrysobalanus icaco et Dialium guineense ou sous forme de touffes éparses, du fait des actions anthropiques (installation des cultures, recherche de bois de chauffe et de construction, etc). Notons aussi que SèméPodji baigne dans un climat soudano guinéen caractérisé par deux saisons sèches (décembre à février et août à septembre) et deux saisons pluvieuses (avril à juillet et octobre à novembre). La température moyenne s'élève environ à $27{ }^{\circ} \mathrm{C}$ avec une moyenne pluviométrique dépassant annuellement 1100 mm (Chabi et Bohoun, 2009).

\section{Présentation et caractéristiques des ouvrages ECOSAN}

Les latrines ECOSAN sont des fosses étanches ou non d'aisance, semi-enterrées ou surélevées avec la particularité de séparer les fèces et les urines. La profondeur de la fouille ne dépasse généralement pas $30 \mathrm{~cm}$ environ en terrain stable. Les parpaings servent de mur d'élévation et ont pour support le radier ou le béton de propreté. La dalle de couverture de la fosse porte un ou plusieurs trou(s) de défécation et d'aération selon le modèle de latrines. Le tuyau d'aération est un empilement vertical de claustra qui surpasse la superstructure de $60 \mathrm{~cm}$. Il est muni d'un grillage anti-mouche. A l'instar de toutes les latrines, on y rencontre aussi une dalle qui supporte l'usager. La superstructure est en matériaux définitifs mais elle peut être aussi en matériaux locaux selon le choix des bénéficiaires; elle est coiffée de feuilles de tôles ou d'une dalle. Les portes sont en bois ou en tôles. Les bidons de stockage d'urine sont protégés par une petite maçonnerie. Notons qu'il existe des latrines ECOSAN à double fosse de type Vietnamien (LEDF) et les latrines ECOSAN à plaque chauffante de type
Tec pan (LEPC) qui sont construites à SèmèPodji. La spécificité des latrines à double fosse de type Vietnamien est que les portes de vidange sont latérales alors qu'au niveau des latrines de type Tec pan, les plaques de vidange situées derrière sont orientées d'un angle de 45 degrés vers le soleil pour augmenter la température et le séchage des fèces dans les fosses.

$\mathrm{Au}$ total, deux catégories d'ouvrages ECOSAN ont été rencontrées dans la commune de Sèmè-Podji à savoir les latrines et les urinoirs (Photo 1). En matière de latrines, il a été répertorié sur le terrain trois types à savoir : les latrines familiales à fosse unique avec plaque chauffante, les latrines familiales à double fosse avec plaques chauffantes et latrines institutionnelles (modules de trois latrines à double fosse avec plaques chauffantes) dans les écoles.

\section{Mécanisme de fonctionnement, d'utilisation et d'entretien des ouvrages ECOSAN}

Les latrines ECOSAN permettent la déviation des urines et des matières fécales. Ces matières fécales sont émises dans des fosses et les urines sont recueillies dans un bidon. La séparation des urines des fèces diminue considérablement l'humidité dans la fosse. Les fosses sont utilisées de manière alternante. Les cheminées de ventilation permettent l'aération de la fosse. Les plaques chauffantes assurent la déshydratation rapide des matières fécales. L'utilisation de la cendre ou de la sciure après chaque défécation permet de réduire davantage l'eau des matières fécales de la fosse ainsi que les odeurs.

L'utilisation des latrines ECOSAN à plaque chauffante est simple. Chaque fois qu'un usager finit de l'utiliser, il verse une quantité déterminée de la cendre ou de la sciure de bois dans la fosse. Cette cendre absorbe l'humidité et augmente la basicité ( $\mathrm{pH}$ élevé) des matières fécales, ce qui facilite la destruction des germes pathogènes. Il en 
résulte que le contenu de la fosse est sec. $\mathrm{Au}$ niveau des latrines à plaque chauffante à double fosse, les trous de défécation sont utilisés de façon alternante c'est-à-dire quand l'un est en service l'autre est fermé. Quant aux bidons d'urine, ils sont régulièrement contrôlés par les bénéficiaires afin qu'ils ne soient remplis et que l'urine ne déborde.

La Figure 2 est une illustration du niveau d'utilisation des ouvrages ECOSAN dans les six (06) arrondissements de la commune de Sèmè-Podji.

Il ressort de l'observation de cette figure que les latrines ECOSAN sont fréquemment utilisées dans les arrondissements de Djrègbé, d'Epkè et SèmèPodji et Agblangandan et peu utilisées à Aholouyèmè, et Tohouè. Notons que le faible taux d'utilisation desdits ouvrages dans ces deux arrondissements serait dû au fait qu'ils sont considérés comme un luxe par certains bénéficiaires au point où seuls le chef de famille et ses épouses ont accès à l'ouvrage. Les autres membres du ménage (enfants et autres) devront se débrouiller dans la nature. A ce facteur, s'ajoutent aussi les pesanteurs sociologiques pour lesquelles, les populations de ces milieux conçoivent mal la valeur qui est désormais accordée à ce « déchet » que sont les fèces et dont l'utilisation sous cette forme constitue un choc moral difficilement acceptable.

\section{Stockage et transport des urines}

Les urines sont directement recueillies dans des bidons de 301 ou 501 . Ces bidons possèdent au niveau de leur ouverture des entonnoirs qui permettent de converger les urines reçues du tuyau. Une fois ce dernier rempli, il est hermétiquement fermé et déposé à un endroit ombragé ou dans un coin de la maison. Toutefois, il faut éviter de les déposer à côté des puits, des jarres, de la cuisine, dans les habitations, pour des raisons d'hygiène et d'esthétique. Les urines sont gardées dans le bidon pendant une durée de 30 à 45 jours avant d'être utilisées dans les champs.

La Photo 2 présente le dispositif permettant de recueillir l'urine issue des latrines ECOSAN.

Les urines sont transportées différemment selon que l'on soit dans un milieu urbain ou rural. En milieu urbain, les urines peuvent être déplacées à l'aide d'une charrette tandis qu'en milieu rural, les bénéficiaires peuvent se servir d'un vélo pour transporter le bidon d'urine des points de production vers les champs pour un stockage de longue durée dans des tonneaux. Que ce soit en ville ou au village, le transport des urines n'exige pas un coût financier élevé. Le stockage de ces urines se fait dans les bidons plastiques illustrés par la Photo 3 .

\section{Stockage et transport des fèces}

La collecte de fèces des latrines ECOSAN à plaque chauffante est une manière d'assurer la bonne hygiénisation avant que les fosses ne soient vidangées. Une fois que la première fosse est pleine, on la laisse pendant le temps qu'il faut pour remplir la deuxième. Le temps de l'hygiénisation est de 6 à 12 mois selon les régions, plus long pour les régions plus humides et plus froides. Quand la deuxième fosse est pleine, il faut évacuer les fèces hygiénisées (Photo 4) de la première fosse. Le contenu est totalement sec et inoffensif. Le produit peut être composté.

Les fèces hygiénisées obtenues à travers ce procédé sont mises dans des sacs à engrais et sont transportées des latrines vers les champs par des engins à deux roues. Ces fèces sont compostées. Toutes les personnes qui ont participé à ces activités, c'est-à-dire de la vidange jusqu'au transport doivent se doucher à grande eau et au savon et laver les tenues qu'elles ont portées. La Photo 5 illustre ces fèces hygiéniques issues des latrines ECOSAN en expérimentation depuis quelques années à Sèmè-Podji. 
O. L. R. AKIYO et al. / Int. J. Biol. Chem. Sci. 7(6): 2214-2232, 2013

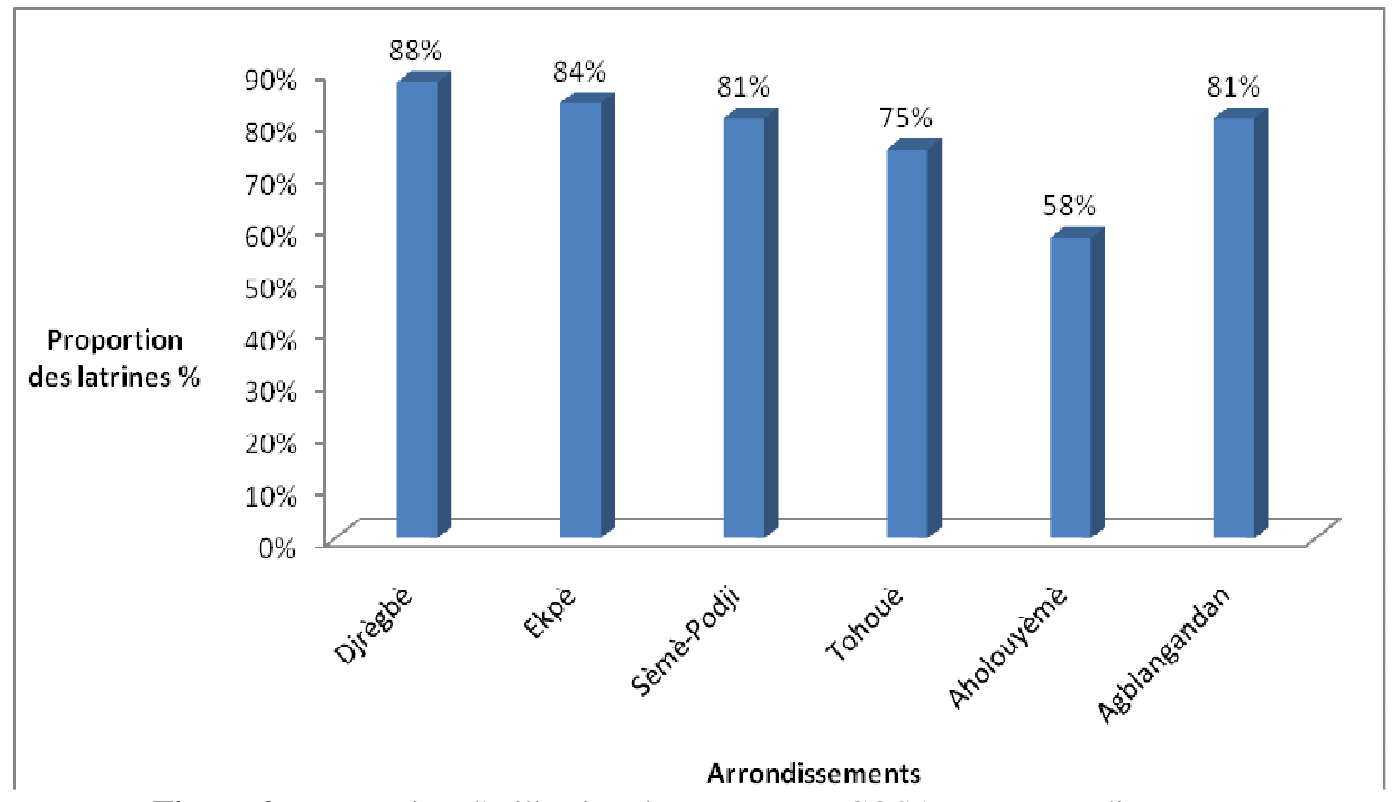

Figure 2 : Proportion d'utilisation des ouvrages ECOSAN par arrondissement. Source : Enquête 2009.

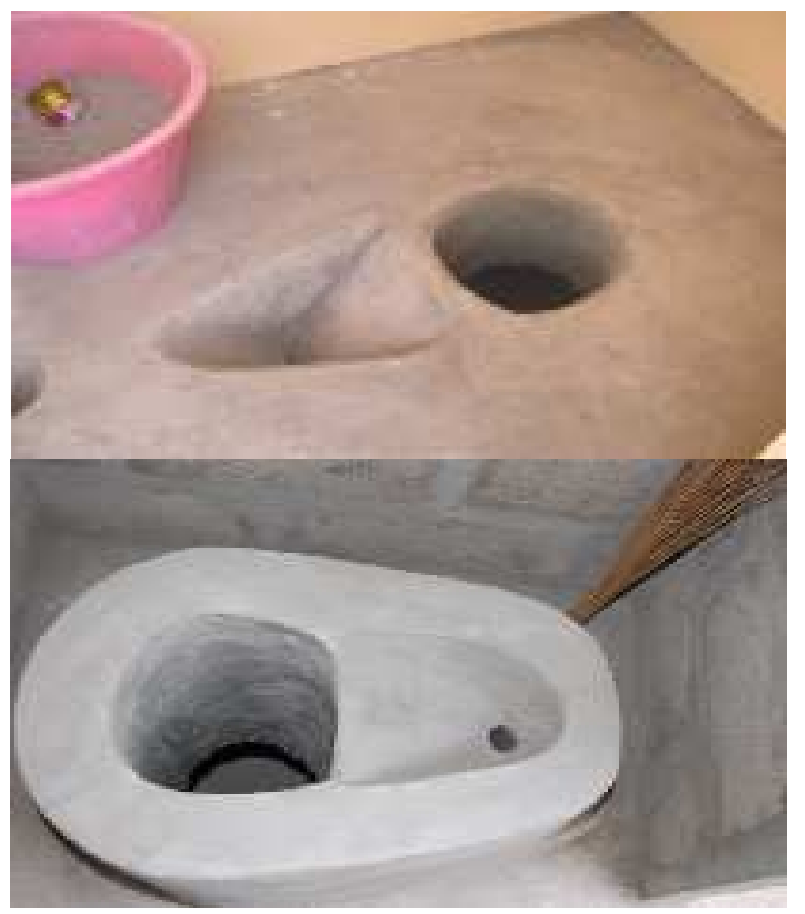

Photo 1 : Deux types de cuvette d'une latrine ECOSAN suivant la position accroupie et assise. Source : CREPA-BENIN, 2006. 


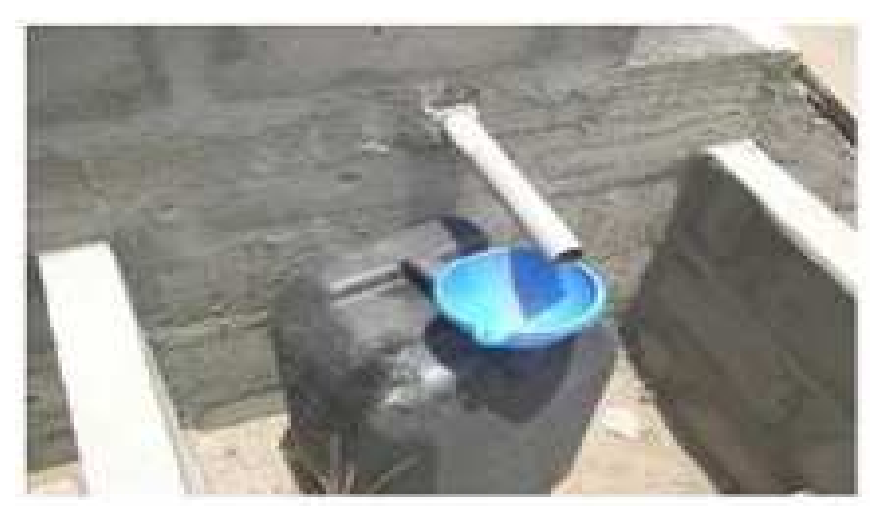

Photo 2 : Emplacement du bidon d'urine des latrines ECOSAN. Source : CREPA-BENIN, ECOSAN 2004.

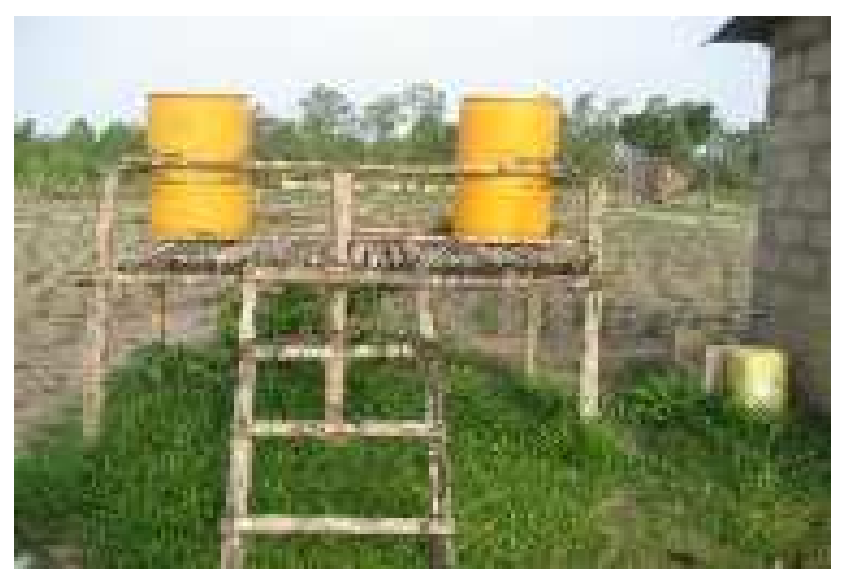

Photo 3 : Stockage d'urine dans le site de maraîchage de VIMAS. Source : CREPA-BENIN, ECOSAN 2009.

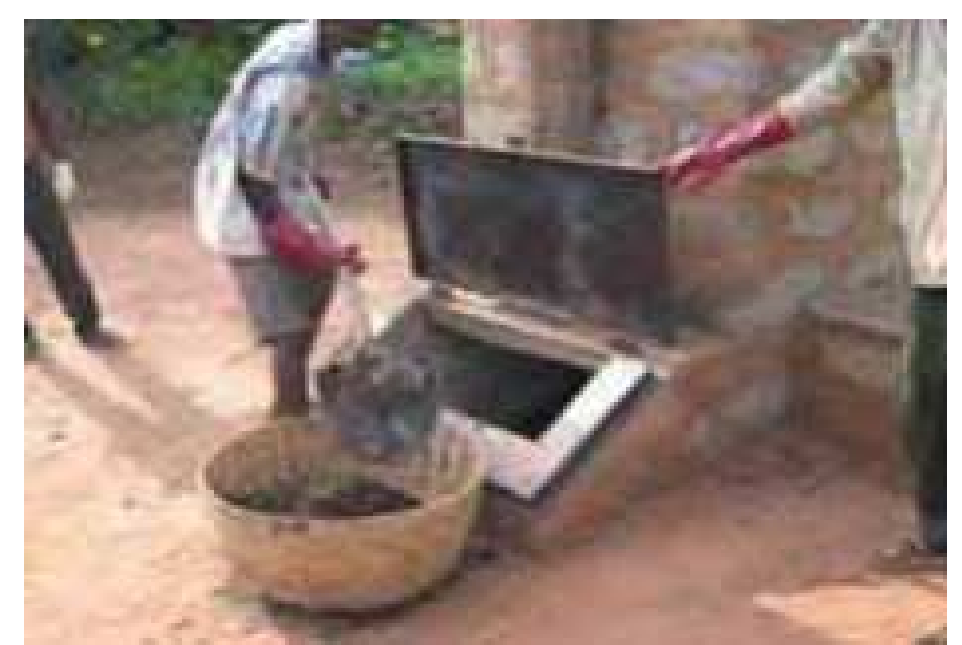

Photo 4 : Séance de vidange de latrine ECOSAN à plaque chauffante dont le produit obtenu sert de compost dans le secteur agricole.

Source : CREPA-BENIN, ECOSAN 2004. 


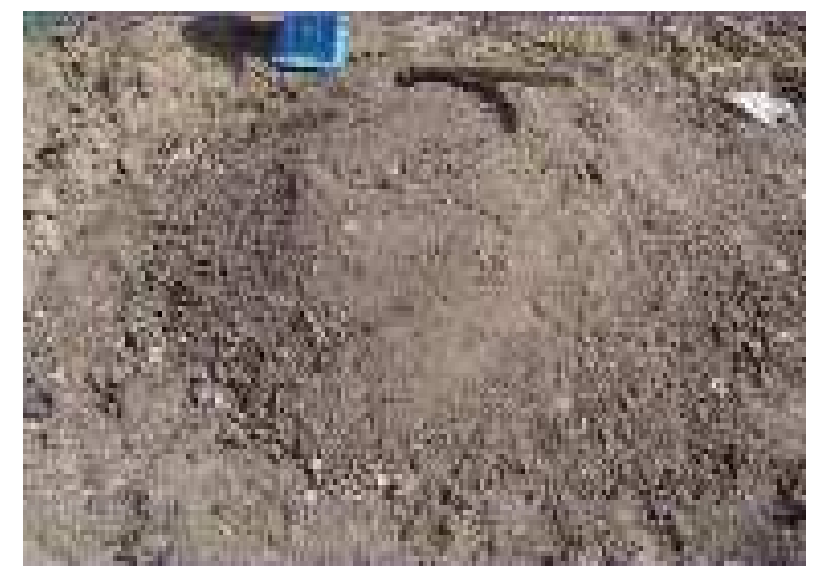

Photo 5 : Tas de fèces hygiéniques servant de compost dans un champ maraîcher à Sèmè-Podji. Source : CREPA-BENIN, ECOSAN 2004.

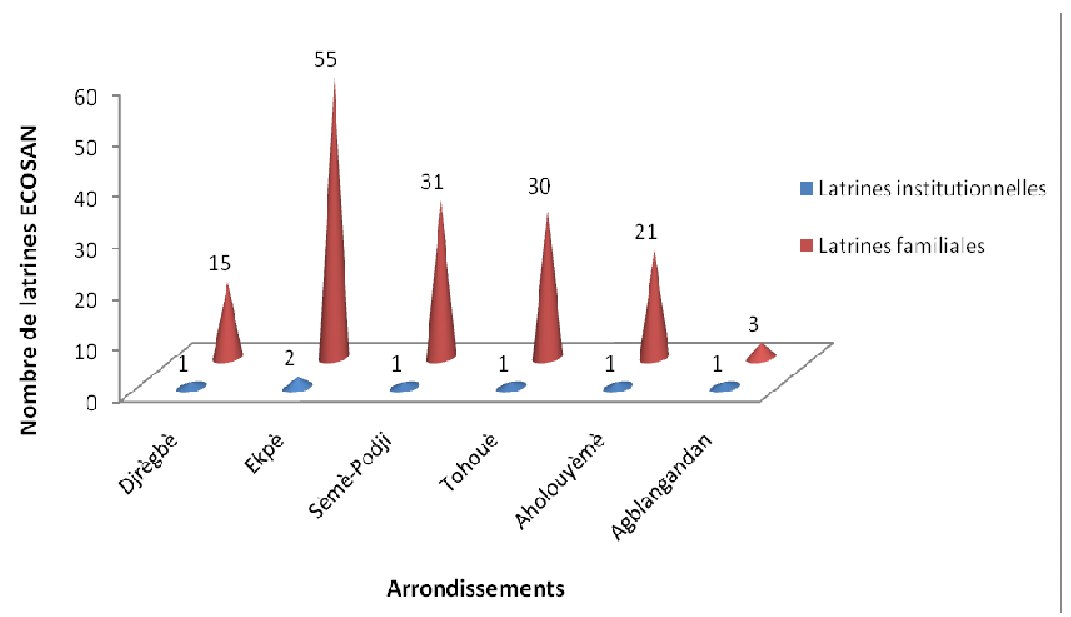

Figure 3 : Répartition de nombre de type de latrines ECOSAN en fonction des arrondissements. Source : Enquête 2009.

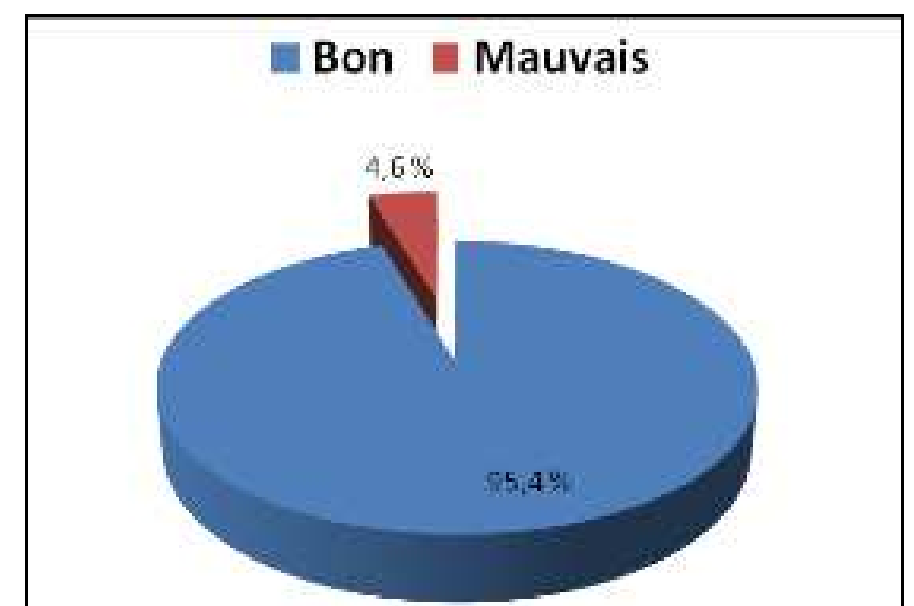

Figure 4 : Perception des bénéficiaires par rapport à l'approche ECOSAN dans la commune de Sèmè-Podji.

Sources : Enquêtes 2009. 


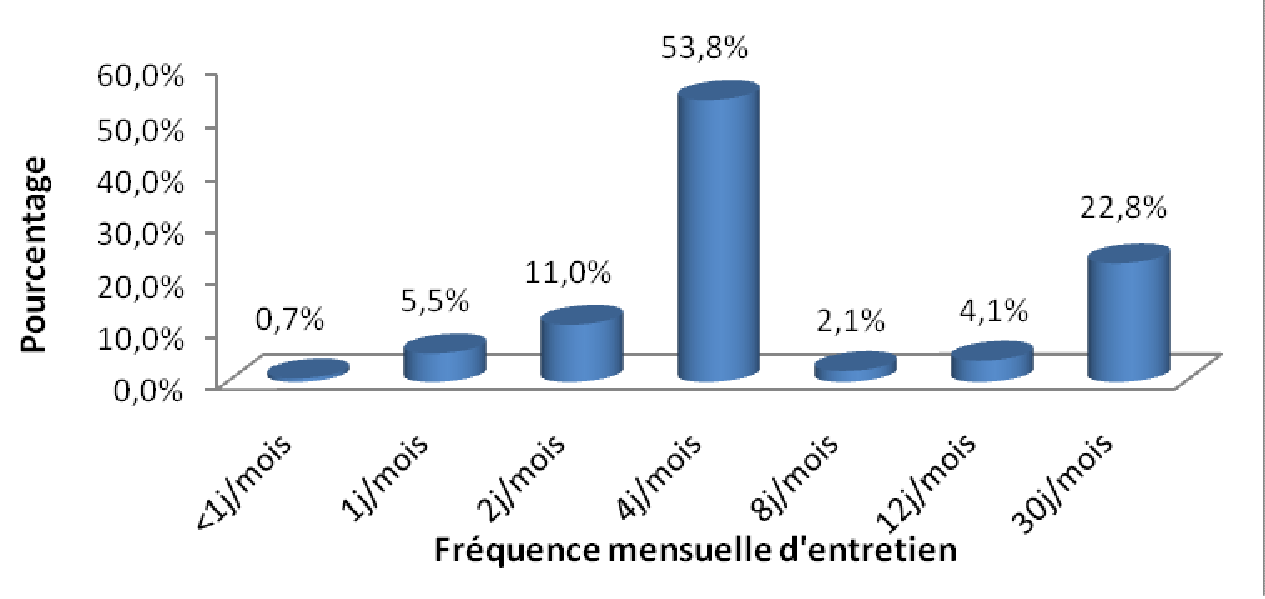

Figure 5 : Pourcentage mensuel d'entretien des ouvrages ECOSAN par les ménages à Sèmè-Podji. Source : Enquêtes 2009.

Tableau 1 : Evolution des différentes affections liées à l'hygiène et l'assainissement dans la commune de Sèmè-Podji 2007 à 2010.

\begin{tabular}{llcccc}
\hline $\mathbf{N}^{\circ}$ & Types d'affections & \multicolumn{4}{c}{ Années et Evolution } \\
\cline { 3 - 5 } & & $\mathbf{2 0 0 8}$ & $\mathbf{2 0 0 9}$ & $\mathbf{2 0 1 0}$ & $\mathbf{2 0 1 1}$ \\
\hline 01 & Paludisme & 2809 & 1622 & 1011 & 854 \\
02 & Choléra & 2052 & 1003 & 827 & 679 \\
03 & Dermatose & 985 & 767 & 453 & 386 \\
04 & Infections respiratoires aiguës & 1463 & 1119 & 728 & 605 \\
05 & Maux de ventre chroniques & 890 & 691 & 409 & 368 \\
06 & Diarrhée & 1028 & 745 & 613 & 431 \\
\hline \multicolumn{2}{l}{ Source : Registre des centres de santé publics et privés des arrondissements de Sèmè-Podji. }
\end{tabular}

\section{DISCUSSION}

Impacts socio-économiques de l'utilisation des latrines ECOSAN

Sur le plan environnemental

Avec l'avènement des latrines ECOSAN dans le milieu, certains enquêtés ont révélé qu'ils ont constaté à un moment donné la réduction de certaines odeurs nauséabondes qui polluaient autrefois leur cadre de vie et le rendait malsain et impropre. C'est dans cette optique, qu'un enquêté d'Agblangandan déclarait ce qui suit «Autrefois, j'avais un accoutrement spécial pour aller me mettre à l'aise dans les latrines traditionnelles et quand j'y sors aussi je marche discrètement pour aller me laver à la douche afin de ne pas indisposer tous ceux que je rencontrais sur mon passage par les odeurs nauséabondes, ce qui n'est plus le cas aujourd'hui avec le système ECOSAN». De plus, cette situation a entraîné par ricochet la diminution de certaines maladies dues à l'hygiène et l'assainissement d'après les agents de santé interrogés à cet effet. Si l'assainissement écologique pouvait être adapté à grande échelle, il protégerait les eaux souterraines, les cours d'eau, les lacs et la mer, de la contamination fécale. Elles offrent aussi une facilité de construction hors sol. L'écosystème est conservé intact puisqu'il n'y a pas d'effet de perturbation. De plus, l'écoassainissement permet d'utiliser l'urine comme 
engrais de haute valeur et de ne pas recourir aux engrais chimiques qui polluent de plus en plus l'écosystème et entraîne des effets négatifs sur la santé des populations (CREPA, 2006). Avec le système de compostage des fèces, les populations de Sèmè-Podji notent, selon leurs déclarations, une réduction du coût des fertilisants et une augmentation du rendement des produits agricoles. Au niveau des arrondissements de Djrègbé, d'Epkè et Sèmè-Podji où cet ouvrage est plus utilisé, 400 à 500 litres d'urines sont produites par chaque individu pendant un an. Ces urines contiennent assez de nutriments (azote, phosphore et potasse) qui permettent de faire pousser 250 kilogrammes de grains, suffisants pour nourrir une personne pendant un an. Elle contient $90 \%$ de valeur en engrais des excréta humains. Pour ce faire, elle doit être diluée avec l'eau et mise directement dans les jardins maraîchers et les champs agricoles ou stockée dans des réservoirs souterrains pour un usage ultérieur. L'installation et surtout l'utilisation et la maintenance de cet ouvrage nécessitent assez de précautions par rapport aux latrines simples. Elles nécessitent alors une formation préalable et un suivi régulier qui nécessitent une certaine expertise.

\section{Sur le plan socioéconomique}

Les latrines écologiques sont de nouveaux modèles d'ouvrages d'assainissement auxquels les populations s'accommodent de plus en plus. Elles sont d'une technologie simple, accessible à tout le monde et très facilement réalisable. Ce sont des latrines peu coûteuses. L'usage de ces ouvrages dans ce milieu dispense plus de $73 \%$ des ménages enquêtés de certaines dépenses en pharmacie relatives aux maladies parasitaires comme le choléra, la diarrhée, etc. Grâce à ECOSAN, les charges financières de la vidange des latrines traditionnelles à l'aide de camion-citerne qui s'élèvent au moins à 50.000 FCFA par semestre sont économisées. Cette économie faite à partir de ce type d'ouvrage depuis la vidange jusqu'à la préservation contre certaines pathologies permet à certains bénéficiaires de Sèmè-Podji d'investir dans d'autres secteurs d'activités comme l'éducation et la nourriture de leurs enfants. De plus, les fèces compostées obtenues après vidange des fosses servent d'engrais naturel et jouent un rôle important dans la réduction d'importantes dépenses liées à l'achat des engrais chimiques dans le domaine agricole et maraîcher avec pour corollaire le développement de certaines maladies. Ces fèces sont également revendues par certains acteurs de la chaîne à d'autres usagers du milieu maraîcher à 2000FCFA le sac de $50 \mathrm{~kg}$; ce qui leur permet aussi de se doter de quelques ressources financières pour subvenir à d'autres besoins de leur ménage.

\section{Sur le plan sanitaire}

Les systèmes d'éco-assainissement, lorsqu'ils sont correctement gérés et entretenus, ne produisent ni mouches, ni autres insectes, ni odeurs nauséabondes car les niveaux d'humidité sont trop faibles pour générer des mouches. Cette situation constitue donc un grand avantage sur les toilettes ordinaires à fosse. Sur le plan sanitaire, les latrines ECOSAN permettent de diminuer les infections liées aux maladies hydro fécales. Ainsi, bon nombre de bénéficiaires de ces types d'ouvrages ont aussi reconnu lors des investigations, qu'avec l'observation des règles d'hygiène qui a commencé dans le milieu avec l'utilisation des latrines ECOSAN, les mouches ont considérablement diminué ainsi que les maladies gastro-intestinales telles que les diarrhées, les maux de ventre, etc. De plus, l'utilisation des fèces compostées à la place des engrais chimiques participe aussi à la réduction de certaines maladies occasionnées par les effets secondaires desdits produits comme l'intoxication alimentaire, les problèmes oculaires, dermiques, neurologiques, respiratoires (Daran, 2004). Les statistiques suivantes recueillies dans les différents centres de santé publics et privés du milieu d'étude viennent confirmer cette position des enquêtés sur l'importance sanitaire de cet ouvrage d'hygiène et d'assainissement qu'est l'ECOSAN. 
Une analyse de l'évolution des différentes maladies présentées dans le Tableau 1 montre une tendance à la baisse. Cette situation serait due, selon les investigations, à la prise en compte et au respect d'un certain nombre de mesures d'hygiène et d'assainissement comme l'usage des latrines ECOSAN et de fèces compostées dans le secteur agricole par les populations de Sèmè-Podji.

\section{Sur le plan agronomique}

Les latrines ECOSAN permettent aux populations de Sèmè-Podji d'obtenir des produits nécessaires pour fertiliser les sols. En effet, les fèces issues de ces ouvrages d'assainissement sont riches en phosphore $(0,97 \%)$ et en carbone organique $(11,2 \%)$. L'urine quant à elle est riche en azote $(7,3$ g/1). Les deux substances provenant de ce processus d'éco-assainissement à savoir l'urine et les fèces jouent un rôle non négligeable dans le domaine agronomique. Pour preuve, l'essai d'urine hygiénisée expérimentée dans ce milieu d'étude a entraîné une grande rentabilité de la production de la grande morelle au Bénin (Kpangon, 2009). A cela s'ajoute la plantation de manguiers, de bananiers, de goyaviers, d'avocatiers, de papayers et des légumes (exemples de cultures de potiron, de tomates, de fruits de la passion) dont les rendements ont été très satisfaisants grâce à l'utilisation de ces fertilisants dans la commune de SèmèPodji. $67 \%$ de la population enquêtée ont reconnu l'importance des fertilisants obtenus à partir du système ECOSAN qui, selon eux, donnent plus de vitalité aux espèces végétales, accélèrent leur croissance et améliorent leur goût une fois à maturité, ce qui n'est pas le cas au niveau des engrais chimiques qui dénaturent les produits agricoles et les font dépenser davantage. C'est pour illustrer ce cas de figure qu'une maraîchère de Tohouè disait : «Chaque fois que j'utilise de l'engrais chimique pour semer le gombo, je constate qu'il est peu gluant et peu succulent alors que depuis que j'ai commencé à utiliser les fertilisants issus d'ECOSAN, j'ai constaté beaucoup d'amélioration car le produit qui y est issu est plus gluant avec un goût intéressant qui attire davantage mon mari et mes enfants ».

\section{Sur le plan politique}

Le Sommet de Johannesburg sur le Développement durable de 2002 a permis de préciser les Objectifs du Millénaire pour le Développement (OMD) en ce qui concerne l'eau et l'assainissement. La promotion des latrines ECOSAN répond aux objectifs de réduction de la pauvreté et à l'atteinte du point 7 des Objectifs du Millénaire pour le Développement tels que fixés avant 2015 intitulé « Assurer un environnement durable ». La promotion de ce système d'assainissement répond également au processus de lutte contre la pauvreté ou l'atteinte du développement durable au Bénin en général et dans le milieu d'étude en particulier. Ainsi, elle limite la prolifération anarchique de certaines pathologies qui ruinent les populations sur les plans physique, moral et financier. D'où l'importance de veiller à une conjugaison des efforts, car nous sommes conscients que l'amélioration de l'assainissement seul ne peut faire le développement mais elle contribue largement au développement (DSCRP3). Adoptées à une grande échelle, ces latrines résolvent bien une partie du récurrent problème de la gestion des excréta, des eaux usées et des déchets en général. Elles dispensent par exemple de la construction des bassins de lagunage, permettant à la commune de disposer de ses fonds pour d'autres projets de développement. Ce sont là autant d'atouts sociopolitiques dont regorgent ces ouvrages qui, de façon majoritaire, sont réalisés au profit de certaines familles et institutions dans la commune de Sèmè-Podji.

Le nombre de latrines ECOSAN familiales réalisées est largement supérieur à celui construit au niveau des institutions. L'arrondissement d'Epkè possède le plus grand nombre d'ouvrages ECOSAN, soit 55 latrines ECOSAN familiales et 2 latrines ECOSAN institutionnelles. L'arrondissement d'Aglangandan est le moins pourvu en 
ouvrages ECOSAN. On n'y dénombre que trois latrines familiales et une latrine institutionnelle. Cette disparité en matière de réalisation de ce type d'infrastructures d'assainissement dans le milieu d'étude est illustrée par la Figure 3.

A l'analyse des résultats, nous remarquons aussi que l'ONG CREPA- BENIN s'est investie plus dans la réalisation de latrines familiales qu'institutionnelles dans le milieu. Cette situation pourrait s'expliquer d'une part par le mécanisme de financement de ces ouvrages. En effet, pour ce qui est des ouvrages ECOSAN de type familial, les ménages bénéficiaires participent pour $20 \%$ du montant global de la réalisation de l'ouvrage ce qui constitue une source de motivation importante pour le CREPA dans l'accord de partenariat pour la réalisation de ces ouvrages. D'autre part, les ouvrages institutionnels ne sont accessibles aux usagers que pendant un temps limité correspondant aux heures d'ouverture et de fermeture de ces institutions. Or, le besoin de se soulager peut subvenir à tout moment de la journée ou de l'année, d'où aussi l'option faite par CREPABENIN de mettre l'accent sur la construction des ouvrages de type familial.

Sur le plan socioculturel, l'utilisation des fèces dans l'agriculture a été acceptée et pratiquée avec quelques réticences au niveau de certains bénéficiaires qui n'ont pas encore cerné les biens fondés de l'approche ECOSAN. Ces derniers conçoivent donc mal l'utilisation de leurs excréta comme fertilisants. Il se pose alors à ce niveau un problème purement psychologique qu'il va falloir régler avec tact pour ne pas heurter des susceptibilités. Mais au-delà de ces quelques résistances, force est de constater que grâce à l'introduction des latrines ECOSAN, des changements quantitatifs et qualitatifs de comportements par rapport aux mauvaises pratiques de gestion des excréta sont manifestement visibles. Le constat laisse paraître un réel changement dans les habitudes en matière de pratiques d'hygiène et d'assainissement.
Notons aussi que l'obtention de ces fertilisants ne concerne pas les immeubles à plusieurs niveaux pour des raisons de mise en place du dispositif de collecte, de traitement et de transport des fèces et des urines.

Selon les statistiques illustrées par la Figure 4, les bénéficiaires sont globalement satisfaits des services fournis par ces ouvrages même si certaines poches de résistance demeurent par endroits pour plusieurs raisons, notamment les pesanteurs socioculturelles. Au total, on note clairement que les latrines ECOSAN répondent aux attentes des bénéficiaires car plus de 95\% apprécient positivement ce système d'éco-assainissement et estiment que cette nouvelle technologie permet de protéger la nappe phréatique et d'avoir un cadre de vie sain et propre. Toutefois, quelques réticences sont émises par une frange des bénéficiaires $(4,6 \%)$. Cette deuxième catégorie d'acteurs souhaite que des améliorations soient apportées par rapport aux dimensions de la cabine, au trou de défécation, au nombre de marches jugées trop important pour pouvoir faciliter l'accès aux personnes âgées, etc. La liste n'est donc pas exhaustive car au-delà des multiples avantages que procurent les ouvrages ECOSAN, ils présentent aussi d'autres contraintes non négligeables.

\section{Quelques contraintes des latrines ECOSAN}

Face aux nombreux avantages qu'offrent les latrines ECOSAN, force est de constater que ces ouvrages présentent aussi de nombreuses contraintes qui freinent sa promotion et auxquelles il urge de trouver des solutions. Au nombre de celles-ci, nous pouvons énumérer :

$\checkmark \quad$ la manipulation des déchets : même stabilisés, les excréta et les urines font l'objet de plusieurs tabous. Les populations n'ont pas l'habitude de les manipuler, des changements de comportements à travers des séances de sensibilisation sont donc nécessaires pour briser les résistances relatives à la promotion de la technologie ; 
$\checkmark \quad$ le niveau des fosses: les fosses surélevées posent quelquefois des difficultés d'accès aux personnes âgées et aux handicapés ;

$\checkmark \quad$ l'utilisation de la cendre : l'ajout de la cendre après chaque défécation, recommandé pour le bon fonctionnement de la latrine est parfois irrégulier par négligence ou par ignorance au niveau des populations ;

l'usage de l'eau : l'eau étant interdite dans la fosse, son utilisation pour le nettoyage anal pose des difficultés aux usagers qui, pour ce faire, doivent se déplacer à l'intérieur de la superstructure ;

$\checkmark \quad$ la nature des matériaux de nettoyage : les matériaux solides de nettoyage anal ne sont pas admis dans la fosse. Il est alors recommandé de les stocker dans un contenant où ils seront brûlés plus tard : une attitude qui est parfois contraignante pour les populations.

Mais au-delà de ces contraintes, il faut noter que les populations de Sèmè-Podji accordent une place importante à l'entretien desdits ouvrages (Chabi et Bohoun, 2009). Cette situation se note clairement à travers la Figure 5 qui montre que la fréquence d'entretien est surtout élevée au niveau des ouvrages ECOSAN des ménages. En effet, selon les investigations, 53,8\% des ménages entretiennent leurs ouvrages 4 jours par mois (soit environ 1 fois par semaine) et 22,8\% font un entretien quotidien des ouvrages. Cette situation montre que les populations adhèrent à la logique de l'amélioration de leur cadre de vie en participant régulièrement à l'entretien des ouvrages. La plupart des ouvrages visités sont relativement bien entretenus. Il faut noter aussi que cette prouesse est due au dynamisme du CREPA-BENIN qui œuvre pour une amélioration du cadre de vie des populations sur le plan de l'hygiène et de l'assainissement à travers non seulement les ouvrages ECOSAN construits, mais également par des sensibilisations et des formations menées par les animateurs endogènes choisis au sein de la population. Ces derniers assurent l'éducation de leurs pairs à l'aide des Kits d'animation qui sont mis à leurs dispositions par CREPABENIN.

$\mathrm{Au}$ total, il convient de noter que contrairement à certaines littératures, ce système s'est avéré satisfaisant au niveau de la commune de Sèmè-Podji même si dans certains pays, des incertitudes planent en ce qui concerne la qualité des excréta et des urines humains.

En effet, plusieurs auteurs (Kpangon, 2009; Kessou, 2000; Tandia, 1996) et structures (CREPA-BENIN, CREPA-SIEGE, $\mathrm{DHAB}$, etc.) ont montré que ces produits qui viennent remplacer les engrais chimiques ne sont pas nocifs à l'homme car les dispositions et la durée de leur déshydratation constituent des mesures de protection des populations contre une quelconque nuisance de ces fertilisants. De plus, il faut noter que les fèces sont traitées par déshydratation qui est favorisée par la chaleur du soleil, la ventilation et l'ajout de matériau sec absorbant (cendre, sciure de bois, sable). La cendre joue un rôle déterminant dans l'hygiénisation des fèces car elle présente trois avantages car elle couvre les fèces et évite les odeurs et les mouches, aide le séchage, ce qui facilite l'augmentation du pH (c'est-à-dire que le contenu devient plus basique avec pour conséquence directe, l'élimination des pathogènes). A travers ce processus, les fèces sont donc traitées et hygénisées (destruction des pathogènes) par déshydratation et augmentation du $\mathrm{pH}$. Notons aussi qu'à défaut de la cendre, les populations peuvent également recourir à la terre sèche, ou de la sciure de bois ou du sable. La contrainte de cette méthode est la lenteur du processus d'hygiénisation (Desconnets, 1997). D'où la nécessité d'attendre au moins 12 mois avant l'utilisation des fèces.

Quant aux urines, même si elles contiennent à l'état initial de l'acide, elles sont bien canalisées vers un récipient et traitées par stockage pendant des semaines, ce qui ne facilite pas l'auto-contamination. En effet, notons que la déshydratation faite à travers la ventilation des cuves, l'ajout de la la matière 
sèche et le déplacement sous la plaque métallique du tas de fèces formé sous le tuyau de chute, constituent des dispositions de quasi-neutralisation des risques sanitaires liés à l'utilisation de ces fertilisants.

En somme, même si nulle part, le risque zéro n'existe au monde, il convient cependant de retenir que les deux types d'excréta, ainsi hygiénisés, ne présentent pas de risque de contamination et peuvent donc être utilisés comme fertilisants en agriculture. L'essentiel est que les dispositions de collecte, de traitement et de transport initialement développées soient scrupuleusement respectées tout au long du processus par les populations.

\section{Quelques approches de solution}

$\mathrm{Au}$ terme de cette étude, il ressort que les problèmes environnementaux dans la plupart des cas nécessitent beaucoup de moyens pour leur résolution. La présente étude nous a permis de mettre en évidence un acteur potentiel dans la promotion de la construction des ouvrages d'assainissement écologique et des comportements favorables à la santé et à l'environnement. Mais pour limiter quelques désagréments de ces ouvrages ECOSAN initialement évoqués, il importe de :

$\checkmark$ augmenter les dimensions de la cabine afin de permettre aux personnes, surtout celles qui sont obèses de se sentir à l'aise ;

$\checkmark$ privilégier les institutions telles que: les écoles, les centres de santé, les lieux publics, etc. dans la réalisation de ces infrastructures d'assainissement ;

$\checkmark$ veiller à la vidange effective des matières fécales après un stockage d'un an tel que recommandé par le CREPA en vue de son utilisation effective pour le compostage ;

$\checkmark$ renforcer le système de sensibilisation des populations sur l'importance et la mise en œuvre effective des mesures relatives à l'utilisation des ouvrages ECOSAN;

$\checkmark$ développer des systèmes d'assainissement écologique appropriés à la demande, abordables et durables dans le contexte local, en collaboration avec les populations bénéficiaires et les autres acteurs de développement;

$\checkmark$ exiger le port des équipements de protection individuelle (EPI) tels que les gants, les bottes, les lunettes, les cache-nez, etc., par les populations lors de la collecte et du transport de ces fertilisants naturels pour minimiser les éventuels risques sanitaires ;

$\checkmark$ créer un environnement institutionnel favorable aux approches novatrices en matière d'assainissement, en intégrant dans le cadre législatif, la politique nationale, le plan de développement de toutes les communes du Bénin le doublon "assainir et recycler";

$\checkmark$ appuyer et promouvoir la participation de l'Etat et du secteur privé local dans la fourniture de services d'assainissement, particulièrement en ce qui concerne les systèmes écologiques.

\section{Conclusion}

Depuis les indépendances, la gestion des eaux usées et des excréta constitue pour les pays africains en général et pour le Bénin en particulier, un véritable problème de santé publique. Aussi, la promotion de l'approche ECOSAN visant à protéger la santé et l'environnement par une hygiénisation des excrétas humains en vue de leur utilisation comme fertilisants dans l'agriculture dans ce milieu d'étude permet d'inclure l'assainissement écologique dans son programme de développement durable. Non seulement ces ouvrages participent de la résolution des problèmes d'hygiène, d'assainissement et de fertilisants des sols qui font partie intégrante de la lutte contre la pauvreté mais encore à l'amélioration des revenus des populations. L'expérience menée par CREPA-BENIN dans la commune de Sèmè-Podji est très reluisante et mérite d'être étendue aux autres localités du Bénin grâce à la contribution de l'Etat et d'autres partenaires (ONGs) même si, quelques pesanteurs sociologiques freinent son élan par endroits.

Il est vrai qu'au-delà d'une technologie spécifique, il s'agit donc plus d'une 
"philosophie" : des matières considérées jusqu'ici comme des déchets à évacuer, désormais appréhendées, au travers de l'utilisation de certaines techniques simples, comme des intrants, entrant dans le cycle de régénération de la production agricole. Il est donc souhaitable de sensibiliser et de former les populations pour une acceptabilité culturelle $\mathrm{du}$ principe de recyclage des excréments afin de parvenir à mettre sur pied un système de collecte de la production qui soit plus viable au niveau socioéconomique et environnemental.

\section{REFERENCES}

Adam KS, Boko M. 1980. Le Bénin. Ed. EDICEF ; 93 p.

Adanhoumè JK. 2000. Pollution des sols, des eaux superficielles et souterraines par les engrais chimiques: Possibilité de lutte contre une telle pollution dans le Sud de la République du Bénin.Mémoire pour l'obtention du DEA - Gestion de l'Environnement, UAC, $63 \mathrm{p}$

Ahokponou DA. 2011. Pollution de la berge ouest du lac Nokoué et du Chenal : problème de santé des populations riveraines à Cotonou. Mémoire de Maitrise ; $90 \mathrm{p}$

Aïssi MJ. 1992. Impact des déchets domestiques sur la qualité de la nappe phréatique à Cotonou. Mémoire d'aménagement et protection de l'environnement, UNB/CPU ,88p

Akiyo R. 2012. L'occupation de la Commune fluvio-lacustre de Sô-Ava: les fondements d'une adaptation originale et d'une mise en valeur d'un environnement spécifique du sud-Bénin. Thèse de Doctorat Unique en Gestion de l'Environnement, FLASH/EDP/UAC, $372 \mathrm{p}$.

Akiyo R. 2006. Environnement lacustre et santé des populations: cas de la commune de Sô-Ava. Mémoire DEAGestion de l'environnement, FLASH/EDP/UAC, 84 p.
Akiyo R. 2010. Etat de l'environnement et santé des populations dans le complexe fluvio-lacustre de Sô-Ava au sud du Bénin, (Bénin, Afrique de l'Ouest in revue scientifique éditée par Maîtrises Professionnelles/FLASH/UAC, Vol. 2, N³, décembre 2010, pp 93-106.

Akiyo R. 2011. Déterminants socioculturels et économiques du phénomène de sédentarisation dans la Commune lacustre de Sô-Ava au sud du Bénin, Afrique de l'Ouest in revue scientifique éditée par Maîtrises Professionnelles/FLASH/UAC, Vol. 3, N5, décembre 2011, pp 76-90.

Alé G, Degbevi X. 2007. Rapport de l'évaluation des besoins et leurs coûts pour l'atteinte des OMD dans le secteur de l'eau et de l'assainissement au Bénin, août 2007.

Biaou G. 2000. Coopérer et Agir Autrement pour un Mieux-être. Les Editions Flamboyant: Cotonou ; 339 p.

Boko M. 1988. Climats et Communautés Rurales du Bénin : Rythmes Climatiques et Rythmes de Développement. Dijon, Centre de Recherches de climatologie. Thèse de Doctorat d'Etat, 608 pages.

Chabi A, Bohoun Th. 2009. Evaluation de l'appropriation de l'assainissement écologique dans la commune de SèmèPodji: cas des ouvrages Ecosan, Université d'Abomey-Calavi, Bénin Licence professionnelle, $58 \mathrm{p}$.

Coulibaly P, Ouedraogo H, Tandia C. 1994. Assainissement stratégique du village de Roumtanga : des latrines à la demande de la population. Info CREPA $\mathrm{N}^{\circ} 6$.

CREPA-BENIN. 2003. Assainissement Ecologique au Bénin ECOSAN. CREPABENIN.

CREPA-BENIN. 2004. Construction des Latrines VIP à Fosse Unique non Maçonnée à Double Cabine. CREPABENIN : Cotonou.

CREPA-SIEGE. 2005. Rapport d'étude sur le gain socioéconomique de l'investissement dans l'assainissement au Bénin, 59p. 
CREPA-BENIN. 2006. Assainissement écologique en Afrique de l'ouest et du centre pour une contribution efficace à l'atteinte des objectifs du millénaire pour le développement. Pays concernés : Bénin, Burkina Faso, Congo, Côte d'Ivoire, Guinée Conakry, Guinée Bissau, Mali, Niger, Sénégal, Togo. Document de projet, 51p.

CREPA-BENIN. 2006. Fiche Technique des Ouvrages d'Approvisionnement en Eau Potable et d'Assainissement (AEPA) (3ième édition). CREPA-BENIN.

CREPA-BENIN. 2007. Projet d'amélioration de l'approvisionnement en eau potable de l'hygiène et de l'assainissement de base dans la commune de Sèmè-Podji, 27p.

CREPA-BENIN. 2008. Rapport général sur l'atelier de validation des documents de l'étude portant diagnostic des systèmes de gestion des excréta dans les zones hydrogéologiques difficiles, le protocole de recherche-action et les TDR de recherche, $80 \mathrm{p}$.

CREPA-BENIN. 2009. Rapport d'étude sur le gain socioéconomique de l'investissement dans l'assainissement au Bénin, 59p.

Daran A. 2004. Les facteurs de risques de santé liés à la manipulation des insecticides de coton dans la commune de Pèrèrè : Approche socio anthropologique. Mémoire pour l'obtention de DEA, UAC, $62 \mathrm{p}$.

Desconnets S. 1997. Gestion durable des excréta et eaux usées en zone péri-urbaine et rurale. Techniques et stratégies mises en œuvre en Afrique de l'Ouest. Série mémento de l'environnement, CREPA, Cotonou ,114p.

Djossou M. 2009. Evaluation des projets de coopération d'eau, d'hygiène et d'assainissement par l'intermédiation des ONG depuis l'avènement de la décentralisation : cas du réseau d'eau de Djrègbé-Aholouyèmè dans la Commune de Sèmè-Podji dans le département de l'Ouémé au Bénin, Mémoire d'ingénieur, Université de Poitier, 35p.
Document WASH. 2004. Eau Assainissement et l'Hygiène, WASH, 10p.

Dossou-yovo AO. 2001. Contribution à la gestion participative et à l'aménagement des zones inondables à Cotonou. Mémoire de DEA, FLASH-UNB, 132p.

Dovonon EF. 2009. La pollution des plans d'eau du Sud Bénin et risques écotoxicologiques: cas du lac Nokoué. Mémoire de DEA/UAC/Bénin, 91p.

Edoun GE. 2008. Aménagement urbain et ses contraintes à Cotonou: cas du lac Nokoué et de la lagune de Cotonou. Mémoire de DEA, FLASH-UNB ,132p.

Gibigaye M. 2002. Impact socio-économique et environnemental de l'introduction des nouvelles technologies agricoles dans le monde rural : cas des coopératives d'utilisation des machines agricoles (CUMA) de Bembèrèkè. Mémoire pour l'obtention du DEA - Gestion de l'Environnement, UAC, $75 \mathrm{p}$.

Gnelé J. 2010. Dynamiques de planification urbaine et perspectives de développement durable à Cotonou (République du Bénin). Thèse de doctorat, FLASHUAC, Bénin, 339p.

Hartemann P. 2001. Approvisionnement en eau et assainissement en milieu tropical. Med. Trop : 210-213.

Info CREPA $\mathrm{N}^{\circ} 21.1998$. Hygiène et concept socioculturels au Burkina-Faso. BurkinaFaso, 22p.

Info CREPA $\mathrm{N}^{\circ}$ 43. 2004. Assainissement Ecologique: amélioration des sols en perspective et revue à mi-parcours du programme de recherche régional. CREPA-Bénin, 30p.

Info CREPA $\mathrm{N}^{\circ}$ 44. 2004. Assainissement liquide en milieu urbain et péri urbain en Afrique de l'Ouest: situation et perspectives. CREPA-BENIN, 30p.

Info CREPA $\mathrm{N}^{\circ}$ 6. 1994. Assainissement stratégique du village de Roumtenga. Burkina-Faso, $23 \mathrm{p}$.

Kessou A. 2000. Contribution à l'étude d'impact des ouvrages d'assainissement de base sur la qualité de la nappe 
phréatique: cas des latrines puisards et fosses septiques dans la ville de Cotonou. Mémoire DEA en Gestion de l'environnement. FLASH/UNB, AbomeyCalavi, Bénin, 113p.

Kissira A. 2005. Activités agricoles et dégradation des ressources naturelles dans la commune de Segbana (Benin) : Impacts sur la santé des populations, Mémoire de DEA Gestion de l'Environnement 2005 EDP- FLASHUniversité d'Abomey-Calavi (Bénin).

Kouchadé M. 2002. Evaluation de la pollution organique et bactériologique due aux excrétas, aux eaux usées et aux déchets solides dans la lagune de Cotonou. Mémoire de fin d'études. APE/CPU/UAC, 65p+annexes.

Kpangon S. 2009. Etude de la rentabilité financière de l'utilisation de l'urine hygiénisée dans la production marâichère: cas de la grande morelle, mémoire du diplôme d'étude approfondie (DEA), FSA, UAC Bénin, 93p.

Lamy M. 1995. L'Eau de la Nature et des Hommes. Rocher, collection Scieteren, Presses universitaires de Bordeaux : Talence (France) ; 237 p.

MEHU. 2012. Agenda de l'Environnement. MEHU : Cotonou, Bénin ; 184p.

Ministère de la Santé DHAB. 2003. Programme National d'Hygiène et d'Assainissement de Base (PNHAB). Ministère de la santé DHAB ; 86p.

N'Bessa B. 1997. Porto-Novo et Cotonou (Bénin): origine et évolution d'un doublet urbain. Thèse d'Etat ès Lettres. Université de Bordeaux Talence, 456 p.
OMS. 1997. La gestion du Fonctionnement et de la Maintenance pour l'Eau et l'Assainissement. OMS.

Ouinakonhan M. 2003. Importance du coton dans l'assolement des exploitations et dans la dégradation du paysage au Bénin : Cas de la commune de Gogounou, Mémoire de l'obtention du Diplôme d'Ingénieur des Travaux, UAC, 54 p.

PNUD. 2006. Extrait du résumé du rapport mondial sur le développement 2006, $513 \mathrm{p}$.

Savina A, Mathys A. 1994. L'alimentation en eau en milieu urbain dans les quartiers peu défavorisés : une question de partage. Programme Alimentaire en Eau et Assainissement, Afrique de l'Ouest, Abidjan.

Tandia CT, Compaoré J. 1996. Financement de l'assainissement en milieu rural: l'expérience du CREPA à Roumtenga, Burkina Faso. In Info CREPA $\mathrm{N}^{\circ} 1$, pp225-230.

UNICEF. 1998. Comment Susciter un Meilleur Comportement en Matière d'Hygiène: Importance des Mécanismes de Changement en Santé Publique. UNICEF : La Haye ; 47p.

UNICEF. 2005. Eau, assainissement et environnement. Rapport année 2005. UNICEF.

Vissin EW, Sintondji LO, Houssou SC. 2010. Etude de la pollution des eaux et de la contamination du Tilapia guineensis $\mathrm{du}$ canal de Cotonou par le plomb, 229p. 